\title{
Low-Dose Aspirin-Induced Gastroduodenal Mucosal Injury in Japanese Patients with Arteriosclerotic Disease
}

\author{
Isamu Tamura ${ }^{1}$, Tsuyoshi Fujita ${ }^{1}$, Hidetaka Tsumura ${ }^{1}$, Yoshinori Morita ${ }^{1}$, \\ Masaru Yoshida ${ }^{1}$, Takashi Toyonaga ${ }^{1}$, Seiichi Hirano ${ }^{2}$, \\ Hideto Inokuchi ${ }^{3}$, Hiromu Kutsumi ${ }^{1}$ and Takeshi Azuma ${ }^{1}$
}

\begin{abstract}
Background We aimed to elucidate the risk factors and preventive factors associated with chronic low-dose aspirin (L-ASA)-induced gastroduodenal mucosal injury in Japanese patients with arteriosclerotic disease.

Methods This retrospective observational study included 400 L-ASA users who underwent upper gastrointestinal endoscopy. We investigated patients' clinical characteristics, including age, peptic ulcer history, concomitant drugs [i.e. gastric agents, antiplatelet drugs, anticoagulants, non-steroidal anti-inflammatory drugs (NSAIDs), corticosteroids], abdominal symptoms, endoscopic findings, and interruption of L-ASA before endoscopy. The severity of gastroduodenal mucosal lesions was evaluated using the modified LANZA score (MLS).

Results Of 400 patients, $249(62 \%)$ and $41(10 \%)$ had gastroduodenal mucosal lesions (MLS $\geq 1)$ and gastroduodenal ulcers, respectively. Peptic ulcer history, abdominal symptoms, proton pump inhibitor (PPI), histamine type 2-receptor antagonists (H2RA), and the cessation of L-ASA before endoscopy were significantly associated with L-ASA-induced gastroduodenal ulcers; the odds ratio (OR) (confidence interval (CI)) was 5.49 (1.82-16.55), 4.56 (1.93-10.75), 0.12 (0.03-0.42), $0.13(0.04-0.40)$ and 0.11 (0.04-0.29), respectively. Moreover, patients having two or more of five factors [i.e. advanced age $(\geq 75)$, anticoagulants, antiplatelet drugs, NSAIDs and corticosteroids] had a significantly higher prevalence of L-ASA-induced gastroduodenal ulcers [OR (CI): 2.39 (1.002-5.69)].

Conclusion Peptic ulcer history, abdominal symptoms and the summation of risk factors increased the risk for L-ASA-induced gastroduodenal ulcers. H2RAs and PPIs were effective for the prevention of L-ASAinduced gastroduodenal ulcers. The cessation of L-ASA before endoscopy might lead to the underestimation of L-ASA-induced gastroduodenal injury.
\end{abstract}

Key words: low-dose aspirin (L-ASA), gastroduodenal ulcers, risk factors, preventive factors, cessation

(Intern Med 49: 2537-2545, 2010)

(DOI: 10.2169/internalmedicine.49.3824)

\section{Introduction}

Low-dose aspirin (L-ASA), commonly defined as 75-325 $\mathrm{mg}$ daily, is used for secondary prevention of ischemic heart disease and ischemic cerebrovascular disease. There are many reports of scientific evidence of the efficiency of LASA (1-5). In Japan, since 2000, treatment with L-ASA has been permitted as a medical service under health insurance for the prevention of thrombus and embolus in patients suffering from ischemic heart disease or ischemic cerebrovascular disease.

The tendency for L-ASA to be prescribed has increased, because the number of patients with advanced age, diabetes and hypertension, which are risk factors for ischemic heart disease, is increasing (6).

${ }^{1}$ Department of Gastroenterology, Kobe University Graduate School of Medicine, Kobe, ${ }^{2}$ Department of Gastroenterology, Miki City Hospital, Miki and ${ }^{3}$ Department of Gastroenterology, Hyogo Cancer Center, Akashi

Received for publication April 16, 2010; Accepted for publication August 18, 2010

Correspondence to Dr. Takeshi Azuma, azumat@med.kobe-u.ac.jp 
On the other hand, it is well known that L-ASA induces gastroduodenal mucosal injury as an adverse effect similar to other non-steroidal anti-inflammatory drugs (NSAIDs). Because the number of L-ASA prescriptions is high, the absolute number of gastroduodenal mucosal injuries should not be ignored even if the frequency of mucosal injury is small. L-ASA is usually needed for a long time, and sometimes other anti-platelet drugs and anticoagulants are coprescribed, leading to an increased risk of L-ASA-induced mucosal injury that may develop as a sudden bleeding peptic ulcer without pain, and it may be fatal.

In Japanese patients, Nakayama et al reported that the prevalence of L-ASA related bleeding peptic ulcers was steadily increasing in association with the increasing prescription of L-ASA for cardiovascular disease and cerebrovascular disease (7). The ACCF/ACG/AHA Expert Consensus in 2008 reported that the use of L-ASA $(75-325 \mathrm{mg}$ per day) was associated with a 2- to 4-fold increase of the risk for upper gastrointestinal (GI) events (8). A recent Japanese multicenter case-control study consisting of 175 patients hospitalized with upper GI bleeding and 347 controls identified from population registries in the same district by telephone interviews reported that the odds ratio of upper GI bleeding among L-ASA users was 7.7 (9).

Although there are few studies on the risk factors for gastroduodenal mucosal injury in patients receiving L-ASA, similar factors identified for other NSAIDs-induced gastroduodenal mucosal injury have been suggested as risk factors for L-ASA-induced gastroduodenal mucosal injury. These factors include a high aspirin dose, history of peptic ulcer or ulcer complication, use of NSAIDs, advanced age, concurrent use of anticoagulants, and the presence of severe disease (10). Some reports have pointed out that concurrent use of antiplatelet drugs is a risk factor (11-13). In Japanese patients, however, the risk factors for L-ASA-induced gastroduodenal mucosal injury have not been fully investigated.

In this retrospective study, we aimed to elucidate the risk factors and preventive factors associated with L-ASAinduced gastroduodenal mucosal injury in Japanese patients receiving L-ASA for arteriosclerotic disease.

\section{Methods}

\section{Patients}

This was a retrospective observational study performed at Kobe University Hospital from December 2005 through November 2006, and Miki City Hospital from January through December 2007 among patients receiving L-ASA for arteriosclerotic disease.

To select L-ASA users, we chose outpatients in the Department of Cardiovascular Disease of Internal Medicine receiving L-ASA during the investigation period, using the medical treatment fee database of each hospital. Among them, patients with cardiovascular disease or cerebrovascular disease, who had been receiving L-ASA regularly for more than one month and had undergone endoscopy, were enrolled in this study as L-ASA users.

We examined the medical records of L-ASA users, and investigated patients' clinical characteristics, including sex, age, underlying disease, peptic ulcer history, concomitant drugs [i.e. gastric agents, anticoagulants (warfarin potassium), antiplatelet drugs (ticlopidine $\mathrm{HCl}$, clopidogrel sulfate), NSAIDs, corticosteroids], the presence of Helicobacter pylori (HP) infection, reason for endoscopy [abdominal symptoms (i.e. reflux symptoms, abdominal pain, indigestion, obvious bleeding signs (hematemesis, tarry stool) or occult bleeding signs (anemia, fecal occult blood positive))], interruption of L-ASA before endoscopic examination, and endoscopic findings. A peptic ulcer history was confirmed by inclusion in the medical records and/or endoscopic findings of gastroduodenal mucosal scars. Drugs that were prescribed regularly during the L-ASA treatment period, including the day of endoscopic examination, were defined as concomitant drugs. Newly prescribed drugs within one week before endoscopic examination were not considered as concomitant drugs, although one patient had been prescribed proton pump inhibitor (PPI) 1 day before endoscopy and was classified into the no gastric agents group (None). According to the concomitant prescription of gastric agents, the patients were divided into five groups: PPI user, histamine type 2-receptor antagonist (H2RA) user, prostaglandin analogue (PA) user, cytoprotective gastric agents (C) user, and None. Patients receiving any cytoprotective gastric agents and co-prescribed PPI, H2RA, or PA were classified as PPI, H2RA, or PA users, respectively. One patient co-prescribed PPI and PA was classified as a PPI user.

HP status was determined by the presence of serum HP IgG antibodies, urea breath test (UBT), or culture using gastric mucosal specimens obtained during an endoscopic examination. It was based on a description in the medical records that the reason for endoscopy was anemia, and was not defined by the hemoglobin value. The doctor in charge of the patient decided whether the cessation of L-ASA would be possible before endoscopy, after taking the patient's underlying disease into consideration. The cessation period of L-ASA before endoscopic examination was 7 days in almost all patients, although one patient had 3-day cessation and one patient had 5-day cessation.

Patients who were considered to have unstable underlying diseases were excluded from this study because their unstable general state might have affected their gastroduodenal mucosa. The exclusion criteria were as follows; patients with unstable ischemic heart disease or with ischemic heart disease after stenting treatment or open-heart surgery (i.e. coronary artery bypass graft) for six months, patients with unstable cerebrovascular disease or within three months after an episode, patients with malignancy within five years after the diagnosis of malignancy, patients after upper gastrointestinal tract, biliary tract or pancreatic disease surgery, patients within one month after any surgery, and patients with serious injury to the liver, kidney or lung. We collected and ex- 
Table 1. Patient Background $(n=400)$

\begin{tabular}{|c|c|c|}
\hline & \multicolumn{2}{|c|}{ total } \\
\hline Sex (male : female) & \multicolumn{2}{|c|}{$250: 150$} \\
\hline Age (years) & \multicolumn{2}{|c|}{71} \\
\hline (range) & \multicolumn{2}{|c|}{$(24-92)$} \\
\hline Hospital (Kobe : Miki) & \multicolumn{2}{|c|}{$182: 218$} \\
\hline & $\mathrm{n}$ & $(\%)$ \\
\hline \multicolumn{3}{|l|}{ Causative disease with L-ASA } \\
\hline ischemic heart disease & 235 & $(59 \%)$ \\
\hline cerebrovascular disease & 96 & $(24 \%)$ \\
\hline arteriosclerosis obliterans & 32 & $(8.0 \%)$ \\
\hline diabetes mellitus & 24 & $(6.0 \%)$ \\
\hline collagen disease & 13 & $(3.0 \%)$ \\
\hline Peptic ulcer history & 40 & $(10 \%)$ \\
\hline Helicobacter pylori infection & 47 & $(12 \%)$ \\
\hline$(+)$ & 29 & $(7.3 \%)$ \\
\hline$(-)$ & 18 & $(4.5 \%)$ \\
\hline unknown & 353 & $(88 \%)$ \\
\hline \multicolumn{3}{|l|}{ Reason for endoscopy } \\
\hline abdominal symptom & 186 & $(47 \%)$ \\
\hline reflux symptom & 46 & $(12 \%)$ \\
\hline abdominal pain & 58 & $(15 \%)$ \\
\hline indigestion & 13 & $(3.3 \%)$ \\
\hline obvious bleeding sign & 24 & $(6.0 \%)$ \\
\hline occult bleeding sign & 45 & $(11 \%)$ \\
\hline screening & 214 & $(53 \%)$ \\
\hline \multicolumn{3}{|l|}{ L-ASA before endoscopy } \\
\hline non-cessation & 183 & $(46 \%)$ \\
\hline cessation & 217 & $(54 \%)$ \\
\hline
\end{tabular}

amined patient data according to the ethics rules of each institution.

\section{Endoscopic findings}

All endoscopic examinations were recorded digitally. The extent of gastroduodenal mucosal injury was expressed in terms of Kobayashi-Mizushima's modified Lanza score (MLS); numerically from zero (no injury) to five (ulcer) for gastric injury or four (ulcer) for duodenal injury (14-17). We defined an ulcer as a mucosal deficit of more than $3 \mathrm{~mm}$ in diameter. The endoscopic findings were evaluated by one experienced endoscopist (I.T.) in a blind manner after collecting all patient data.

\section{Statistical analysis}

To evaluate differences in the prevalence of injury, we used the $\chi^{2}$ test or Fisher's exact probability test. To identify the factors associated with gastroduodenal ulcers, multiple logistic regression analysis was performed and the odds ratio (OR) and 95\% confidence interval (CI) were obtained. We compared the MLS using the Mann-Whitney test. All analyses were performed using the STATA/SE statistical software system version 10 (StataCorp., College Station, TX, USA). $\mathrm{p}<0.05$ was defined as significant.

\section{Results}

\section{Patient characteristics}

During the investigation period, cardiologists at each hospital prescribed L-ASA (enteric-coated or buffered) to 3,311 patients (1,341 patients at Kobe University Hospital and 1,970 patients at Miki City Hospital). Upper gastrointestinal endoscopy was performed in 957 of the 3,311 patients (29\%). After selection according to inclusion and exclusion criteria, 400 patients (12\%) were finally enrolled in this study.

Patient background is shown in Table 1, 2. Of 400 patients, 250 (62\%) were men (mean age, 70 years old) and $150(38 \%)$ were women (mean age, 72 years old). As the causative disease of L-ASA use, ischemic heart disease was present in 235 patients (59\%), cerebrovascular disease in 96 patients (24\%), arteriosclerosis obliterans (ASO) in 32 patients $(8.0 \%)$, diabetes mellitus (DM) in 24 patients $(6.0 \%)$, and collagen disease in 13 patients $(3.0 \%)$.

Forty patients $(10 \%)$ had a past history of peptic ulcer. In 35 patients $(88 \%)$, peptic ulcer history was confirmed by both a description in the medical records and the endoscopic findings of gastroduodenal mucosal scars. In 5 patients, a peptic ulcer history ( 2 gastric ulcers and 3 duodenal ulcers) was confirmed by inclusion in the medical records only.

In 128 patients (32\%), no gastric agents were coprescribed, while co-prescription of cytoprotective gastric agents, PA, H2RAs and PPIs, was observed in 67 (17\%)( i. e. Teprenone in 40 (10\%), Rebamipide in 15 (3.9\%), Sucralfate hydrate in $5(1.3 \%)$, and others in $7(1.8 \%))$, one $(0.25 \%), 124(31 \%)$ [i.e. quarter-dose H2RAs in $7(1.7 \%)$, half-dose H2RAs in 63 (15.8\%), and full-dose H2RAs in 54 $(13.5 \%)$ ], and $80(20 \%)$ [i.e. half-dose PPIs in $34(8.5 \%)$, and full-dose PPIs in $46(11.5 \%)]$ patients, respectively. Twenty-eight patients received PPI and cytoprotective gastric agents, and 35 patients received H2RA and cytoprotective gastric agents simultaneously. Co-prescription of anticoagulants, anti-platelets, NSAIDs, and corticosteroids was observed in 46 (12\%), 41 (10\%), 29 (7.3\%), and $36(9.0 \%)$ patients, respectively. HP infections were investigated in 47 patients (12\%), 29 of which (62\%) were found to be HP positive. Five of $18 \mathrm{HP}$-negative patients had a history of HP eradication therapy.

Regarding the reason for endoscopy, 186 patients (47\%) had abdominal symptoms; reflux symptoms, abdominal pain, indigestion, obvious bleeding signs, and occult bleeding signs were observed in $46(12 \%), 58(15 \%), 13(3.3 \%), 24$ $(6.0 \%)$, and $45(11 \%)$ patients, respectively. Of 45 patients 
Table 2. Concomitant Drugs $(n=400)$

\begin{tabular}{|c|c|c|c|}
\hline & \multicolumn{3}{|c|}{ total } \\
\hline & $\mathrm{n}$ & & $(\%)$ \\
\hline \multicolumn{4}{|l|}{ Gastric agents } \\
\hline None & 128 & & $(32 \%)$ \\
\hline Cytoprotective gastric agent & 67 & & $(17 \%)$ \\
\hline $\mathrm{PA}$ & 1 & & $(0.25 \%)$ \\
\hline H2RA & 124 & & $(31 \%)$ \\
\hline quarter-dose H2RA & & 7 & $(1.7 \%)$ \\
\hline half-dose H2RA & & 63 & $(15.8 \%)$ \\
\hline full-dose H2RA & & 54 & $(13.5 \%)$ \\
\hline PPI & 80 & & $(20 \%)$ \\
\hline half-dose PPI & & 34 & $(8.5 \%)$ \\
\hline full-dose PPI & & 46 & $(11.5 \%)$ \\
\hline \multicolumn{4}{|l|}{ Other Concomitant drugs } \\
\hline anticoagulant & 46 & & $(12 \%)$ \\
\hline \multicolumn{4}{|l|}{ warfarin } \\
\hline antiplatelet drug & 41 & & $(10 \%)$ \\
\hline ticlopidine & & 37 & $(9.3 \%)$ \\
\hline clopidogrel & & 4 & $(1.0 \%)$ \\
\hline NSAIDs & 29 & & $(7.3 \%)$ \\
\hline non-selective & & 25 & $(6.3 \%)$ \\
\hline selective inhibiotr of $\mathrm{COX}-2$ & & 4 & $(1.0 \%)$ \\
\hline Corticosteroid & 36 & & $(9.0 \%)$ \\
\hline \multicolumn{4}{|l|}{ Abbreviations: } \\
\hline \multicolumn{4}{|c|}{ L-ASA: low-dose aspirin, C: Cytoprotective gastric agents } \\
\hline \multicolumn{4}{|c|}{ H2RA: histamine type 2-receptor antagonists, PPI: proton pump inhibitors } \\
\hline \multicolumn{4}{|c|}{ PA: prostaglandin analogues, NSAIDs: non-steroidal anti-inflammatory drugs } \\
\hline COX: cyclo-oxygenase & & & \\
\hline
\end{tabular}

with occult bleeding signs, the mean hemoglobin level of 35 patients who underwent endoscopy for the examination of anemia was $9.9 \mathrm{mg} / \mathrm{dL}$ (4.4-15.8). Two hundred fourteen patients $(53 \%)$ underwent endoscopy for screening. Before the endoscopic examination, L-ASA administration was interrupted in 217 patients (54\%) to decrease the risk of endoscopy complications, such as bleeding after biopsy.

\section{Prevalence of gastroduodenal mucosal injury}

The MLS for gastric mucosal lesions was 0 in 157 (39\%), 1 in $95(24 \%), 2$ in $36(9.0 \%), 3$ in $28(7.0 \%), 4$ in 52 $(13 \%)$, and 5 in $32(8.0 \%)$, and the MLS for duodenal mucosal lesions was 0 in $365(91 \%), 1$ in $14(3.5 \%), 2$ in 7 $(1.8 \%), 3$ in $2(0.50 \%)$, and 4 in $12(3.0 \%)$.

Of 400 patients, $243(61 \%), 35(8.8 \%)$, and $249(62 \%)$ had gastric mucosal lesions, duodenal mucosal lesions, and gastroduodenal mucosal lesions (MLS $\geq 1$ ), respectively. Thirty-two (8.0\%), $12(3.0 \%)$, and $41(10 \%)$ patients had gastric ulcers $(\mathrm{MLS}=5)$, duodenal ulcers $(\mathrm{MLS}=4)$, and gastroduodenal ulcers, respectively. Gastric mucosal lesions (MLS $\geq 1$ ) were significantly more frequently observed than duodenal mucosal lesions (MLS $\geq 1)$ (61\% vs $8.8 \%$; $\mathrm{p}=$ $0.005)$. Gastric ulcers (MLS=5) were more frequently ob- served than duodenal ulcers (MLS=4), but the difference was not statistically significant $(8.0 \%$ vs $3.0 \%$; $\mathrm{p}=0.062)$.

\section{Bivariate analysis and multiple logistic regression analysis}

To identify the factors associated with L-ASA-induced gastroduodenal ulcers, bivariate analysis using the $\chi^{2}$ test or Fisher's exact probability test, and multiple logistic regression analysis were performed. The possible risk and preventive factors included sex, advanced age, peptic ulcer history, concomitant anticoagulants, concomitant antiplatelet drugs, concomitant NSAIDs, concomitant corticosteroids, presence of abdominal symptoms, concomitant gastric agents, and cessation of L-ASA before endoscopy.

In bivariate analysis, peptic ulcer history, the presence of abdominal symptoms, cytoprotective gastric agents, H2RA, and cessation of L-ASA were significantly associated with L-ASA-induced gastroduodenal ulcers. PPI was significantly associated with L-ASA-induced gastric ulcers. Patients with two or more of five factors [i.e. advanced age $(\geq 75)$, concomitant anticoagulants, concomitant antiplatelet drugs, concomitant NSAIDs, and concomitant corticosteroids] had a significantly higher frequency of L-ASA-induced gastroduo- 
Intern Med 49: 2537-2545, 2010 DOI: 10.2169/internalmedicine.49.3824

Table 3. Bivariate Analysis of the Factors Associated with Gastroduodenal Ulcers in Patients Receiving L-ASA

\begin{tabular}{|c|c|c|c|c|c|c|c|c|c|}
\hline \multirow{2}{*}{\multicolumn{2}{|c|}{ factors }} & & \multirow[b]{2}{*}{$\mathrm{n} / \mathrm{n}$} & \multicolumn{2}{|c|}{$\mathrm{G}$} & \multicolumn{2}{|l|}{$\mathrm{D}$} & \multicolumn{2}{|c|}{$G \vee D$} \\
\hline & & & & $\% / \%$ & $\mathrm{p}$-value & $\% / \%$ & $\mathrm{p}$-value & $\% / \%$ & $\mathrm{p}$-value \\
\hline Sex & & male / female & $250 / 150$ & $7.6 \% / 8.7 \%$ & 0.70 & $4.0 \% / 1.3 \%$ & 0.22 & $11 \% / 9.3 \%$ & 0.64 \\
\hline \multirow[t]{3}{*}{ Advanced age } & (70yr) & $70 \leq /<70$ & $245 / 155$ & $8.2 \% / 7.7 \%$ & 0.88 & $3.3 \% / 2.6 \%$ & 0.77 & $11 \% / 9.7 \%$ & 0.76 \\
\hline & (75yr) & $75 \leq /<75$ & $165 / 235$ & $9.1 \% / 7.2 \%$ & 0.50 & $3.6 \% / 2.6 \%$ & 0.53 & $12 \% / 8.9 \%$ & 0.30 \\
\hline & (80yr) & $80 \leq /<80$ & $70 / 330$ & $8.6 \% / 7.9 \%$ & 0.85 & $4.3 \% / 2.7 \%$ & 0.45 & $11 . \% / 10 \%$ & 0.72 \\
\hline \multicolumn{2}{|c|}{ Peptic ulcer history } & $(+) /(-)$ & 40 / 360 & $15 \% / 7.2 \%$ & 0.085 & $10 \% / 2.2 \%$ & $0.024^{*}$ & $23 \% / 8.9 \%$ & $0.007^{* *}$ \\
\hline \multicolumn{2}{|l|}{$\mathrm{C}$} & $(+) /(-)$ & $67 / 333$ & $15 \% / 6.6 \%$ & $0.022^{*}$ & $3.0 \% / 3.0 \%$ & 1.00 & $18 \% / 8.7 \%$ & $0.023^{*}$ \\
\hline \multicolumn{2}{|l|}{ H2RA } & $(+) /(-)$ & $124 / 276$ & $3.2 \% / 10 \%$ & $0.017^{*}$ & $1.6 \% / 3.6 \%$ & 0.36 & $4.8 \% / 13 \%$ & $0.017^{*}$ \\
\hline \multicolumn{2}{|l|}{ PPI } & $(+) /(-)$ & $80 / 320$ & $2.5 \% / 9.4 \%$ & $0.040^{*}$ & $2.5 \% / 3.1 \%$ & 1.00 & $5.0 \% / 12 \%$ & 0.099 \\
\hline \multicolumn{2}{|c|}{ Anticoagulant } & $(+) /(-)$ & $46 / 354$ & $11 \% / 7.6 \%$ & 0.40 & $2.2 \% / 3.1 \%$ & 1.00 & $13 \% / 9.9 \%$ & 0.51 \\
\hline \multicolumn{2}{|c|}{ Antiplatelets } & $(+) /(-)$ & $41 / 359$ & $12 \% / 7.5 \%$ & 0.36 & $2.4 \% / 3.1 \%$ & 1.00 & $15 \% / 9.7 \%$ & 0.33 \\
\hline \multicolumn{2}{|c|}{ NSAIDs } & $(+) /(-)$ & $29 / 371$ & $17 \% / 7.3 \%$ & 0.070 & $3.4 \% / 3.0 \%$ & 0.60 & $21 \% / 9.4 \%$ & 0.054 \\
\hline \multicolumn{2}{|c|}{ Corticosteroids } & $(+) /(-)$ & $36 / 364$ & $8.3 \% / 8.0 \%$ & 1.00 & $0.0 \% / 3.3 \%$ & 0.61 & $8.3 \% / 10 \%$ & 1.00 \\
\hline \multicolumn{2}{|c|}{ Reason for endoscopy } & abdominal symptom $(+) /(-)$ & $186 / 214$ & $11 \% / 5.1 \%$ & $0.024^{*}$ & $5.4 \% / 0.93 \%$ & $0.015^{*}$ & $16 \% / 5.6 \%$ & $0.001^{* *}$ \\
\hline \multicolumn{2}{|l|}{ L-ASA } & cessation / non-cessation & $217 / 183$ & $1.8 \% / 15 \%$ & $<0.001^{* * *}$ & $0.92 \% / 5.4 \%$ & $0.015^{*}$ & $2.7 \% / 19 \%$ & $<0.001^{* * *}$ \\
\hline \multicolumn{2}{|c|}{ Summation of the factors } & $2 \leq /<2$ & $67 / 333$ & $16 \% / 6.3 \%$ & $0.005^{* *}$ & $3.0 \% / 3.0 \%$ & 1.00 & $19 \% / 8.4 \%$ & $0.007 * *$ \\
\hline \multicolumn{10}{|c|}{ Abbreviations: } \\
\hline \multicolumn{10}{|c|}{ L-ASA: low-dose aspirin, C: Cytoprotective gastric agents } \\
\hline \multicolumn{10}{|c|}{ H2RA: histamine type 2-receptor antago nists, PPI: proton pump inhibitors } \\
\hline \multicolumn{10}{|c|}{ NSAIDs: non-steroidal anti-inflammatory drugs } \\
\hline \multicolumn{10}{|c|}{ G: gastric ulcer, D: duodenal ulcer, GVD: gastric or duodenal ulcer } \\
\hline${ }^{*} \mathrm{p}<0.05,{ }^{* *} \mathrm{p}<0$ & $01,{ }^{* * *} \mathrm{p}<$ & 0.001 & & & & & & & \\
\hline
\end{tabular}

denal ulcers than patients with one or fewer of the five factors (Table 3).

In multiple logistic regression analysis, a peptic ulcer history, presence of abdominal symptoms, H2RA, PPI, and cessation of L-ASA were significantly associated with L-ASAinduced gastroduodenal ulcers; the OR (CI) were 5.49 (1.82$16.55), 4.56$ (1.93-10.75), 0.13 (0.04-0.40), 0.12 (0.03-0.42) and 0.11 (0.04-0.29), respectively. Another multiple logistic regression analysis, with more than two of five factors, was assumed as one of the explanatory variables, patients with two or more of five factors [i.e. advanced age $(\geq 75)$, concomitant anticoagulants, concomitant antiplatelet drugs, concomitant NSAIDs and concomitant corticosteroids] were significantly associated with L-ASA-induced gastroduodenal ulcers [OR (CI): 2.39 (1.002-5.69)] (Table 4).

\section{Comparison between the L-ASA cessation group and the L-ASA non-cessation group}

We compared the clinical characteristics between the LASA cessation group and the L-ASA non-cessation group. In the L-ASA cessation group, significantly more patients were enrolled in Miki City Hospital [182 (84\%) in Miki City Hospital vs $35(16 \%)$ in Kobe University Hospital; p< 0.001]. Patients with a peptic ulcer history were significantly more frequently observed in the L-ASA non-cessation group than in the L-ASA cessation group [28 (15\%) in noncessation group vs $12(5.5 \%)$ in cessation group; $\mathrm{p}=0.001]$.
Also, there was a significant difference in the reasons for endoscopy between the two groups. In the L-ASA cessation group, patients with obvious bleeding signs were significantly less frequently observed than in the L-ASA noncessation group [3 (1.4\%) in cessation group vs $21(11 \%)$ in non-cessation group; $\mathrm{p}<0.001]$.

Table 5 shows the details of the MLS of gastric mucosal lesions in the L-ASA cessation and L-ASA non-cessation groups. The MLS for gastric mucosal lesions in the L-ASA cessation group was significantly smaller than in the L-ASA non-cessation group (1.28 vs $1.87 ; \mathrm{p}=0.0068)$. In PPI and $\mathrm{H}$ 2RA users, although the MLS for gastric mucosal lesions in the L-ASA cessation group was smaller than in the L-ASA non-cessation group, there was no statistical significance ( 0.84 vs 1.02 ; $\mathrm{p}=0.45$ with PPI, 1.05 vs $1.39 ; \mathrm{p}=0.38$ with $\mathrm{H}$ 2RA). In the L-ASA non-cessation group, the MLS of both PPI users and H2RA users for gastric mucosal lesions were significantly lower than in the no gastric agent group (1.02 vs $2.48 ; \mathrm{p}=0.002$ with PPI, 1.39 vs $2.48 ; \mathrm{p}=0.012$ with $\mathrm{H} 2$ RA). There was no significant difference in the MLS between cytoprotective gastric agent users and the no gastric agent group (2.87 vs $2.48 ; \mathrm{p}=1.00$ ). In the L-ASA cessation group, although the MLS of both PPI users and H2RA users for gastric mucosal lesions were similarly lower than in the no gastric agent group, there was no statistical significance (0.84 vs $1.49 ; \mathrm{p}=0.13$ with PPI, 1.05 vs $1.49 ; \mathrm{p}=0.50$ with $\mathrm{H}$ 2RA); however, the prevalence of gastric mucosal lesions 
Table 4. Multivariate Analysis of the Factors Associated with Gastroduodenal Ulcers in Patients Receiving L-ASA

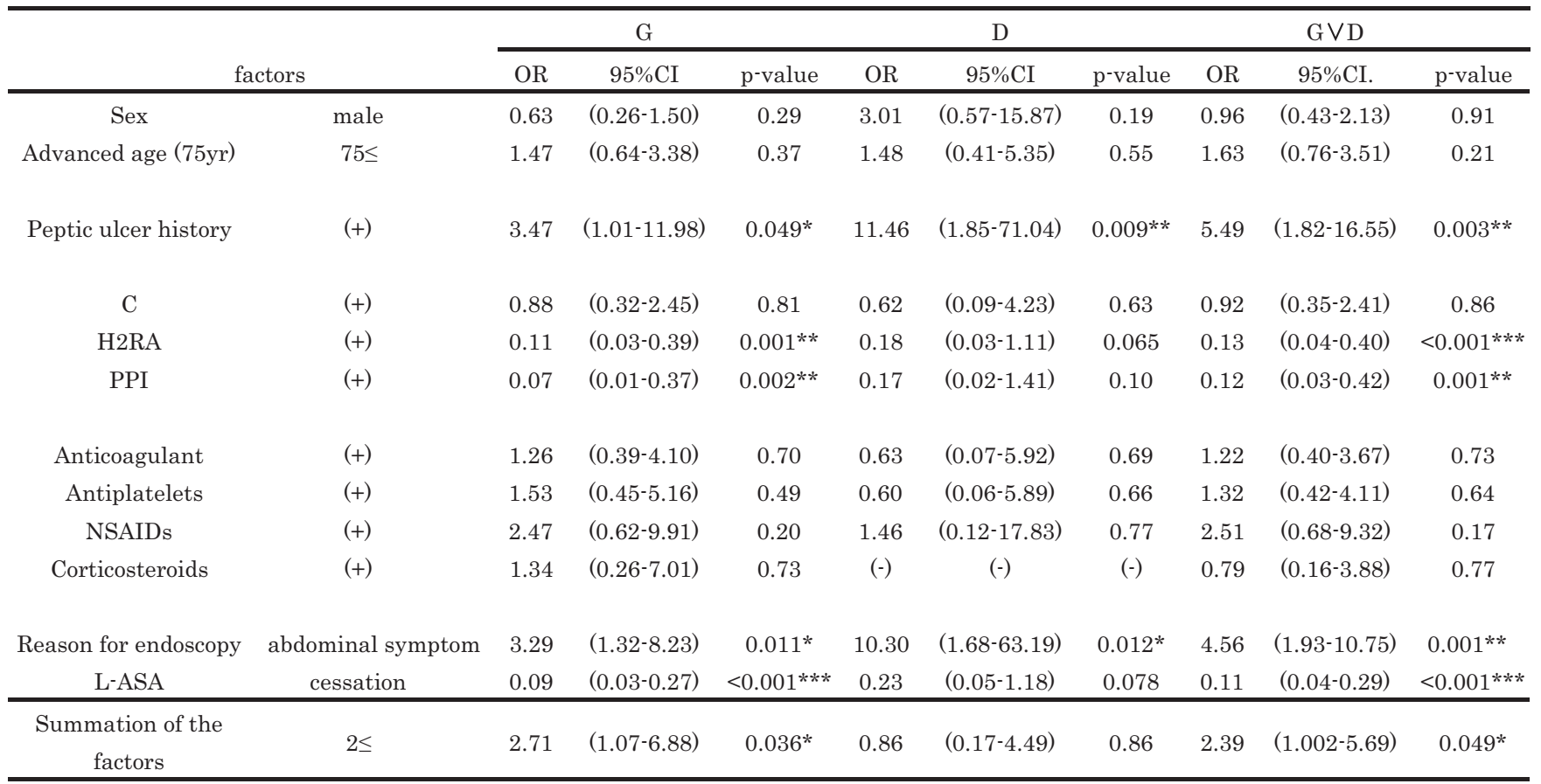

Abbreviations:

L-ASA: low-dose aspirin, C: Cytoprotective gastric agents

H2RA: histamine type 2-receptor antagonists, PPI: proton pump inhibitors

NSAIDs: non-steroidal anti-inflammatory drugs

G: gastric ulcer, D: duodenal ulcer, GVD: gastric or duodenal ulcer

CI: confidence interval, OR: odds ratio

${ }^{*} \mathrm{p}<0.05, * * \mathrm{p}<0.01, * * * \mathrm{p}<0.001$

Table 5. Gastric Mucosal Injury Classified according to MLS in the L-ASA Cessation Group and the L-ASA Non-cessation Group $(n=400)$

\begin{tabular}{|c|c|c|c|c|c|c|c|c|c|}
\hline \multirow{2}{*}{\multicolumn{2}{|c|}{ L-ASA before endoscopy }} & \multicolumn{8}{|c|}{ MLS (stomach) } \\
\hline & & total & 0 & 1 & 2 & 3 & 4 & 5 & Mean scores \\
\hline \multirow{8}{*}{ cessation } & gastric agents & $\mathrm{n}=217(54 \%)$ & $91(42 \%)$ & $60(27 \%)$ & $21(9.7 \%)$ & $9(4.2 \%)$ & $32(15 \%)$ & $4(1.8 \%)$ & $1.28^{* * *}$ \\
\hline & None & $76(35 \%)$ & $27(36 \%)$ & $22(29 \%)$ & $8(11 \%)$ & $3(4.0 \%)$ & $14(18 \%)$ & $2(2.6 \%)$ & 1.49 \\
\hline & $\mathrm{C}$ & $36(17 \%)$ & $11(31 \%)$ & $10(28 \%)$ & $3(8.3 \%)$ & $2(5.6 \%)$ & $9(25 \%)$ & $1(2.8 \%)$ & 1.75 \\
\hline & $\mathrm{PA}$ & $0(0.0 \%)$ & & & & & & & $\mathrm{n} / \mathrm{a}$ \\
\hline & H2RA & $62(28 \%)$ & $26(42 \%)$ & $20(32 \%)$ & $8(13 \%)$ & $3(4.8 \%)$ & $5(8.1 \%)$ & $0(0.0 \%)$ & 1.05 \\
\hline & [quarter/half/full] & [1/33/28] & [1/12/13] & {$[0 / 12 / 8]$} & {$[0 / 4 / 4]$} & {$[0 / 1 / 2]$} & {$[0 / 4 / 1]$} & {$[0 / 0 / 0]$} & [0.00/1.18/0.93] \\
\hline & PPI & $43(20 \%)$ & $27(63 \%) * *$ & $8(19 \%)$ & $2(4.7 \%)$ & $1(2.3 \%)$ & $4(9.3 \%)$ & $1(2.3 \%)$ & 0.84 \\
\hline & [half/full] & {$[21 / 22]$} & {$[12 / 15]$} & {$[4 / 4]$} & {$[1 / 1]$} & {$[1 / 0]$} & {$[2 / 2]$} & [1/0] & [1.05/0.641] \\
\hline \multirow{8}{*}{ non-cessation } & gastric agents & $\mathrm{n}=183(46 \%)$ & $66(36 \%)$ & $35(19 \%)$ & $15(8.2 \%)$ & $19(10 \%)$ & $20(11 \%)$ & $28(15 \%)$ & 1.87 \\
\hline & None & $52(28 \%)$ & $15(29 \%)$ & $7(13 \%)$ & $4(7.7 \%)$ & $4(7.7 \%)$ & $8(15 \%)$ & $14(27 \%)$ & 2.48 \\
\hline & $\mathrm{C}$ & $31(17 \%)$ & $6(19 \%)$ & $2(6.5 \%)$ & $4(13 \%)$ & $6(19 \%)$ & $4(13 \%)$ & $9(29 \%)$ & 2.87 \\
\hline & $\mathrm{PA}$ & $1(0.55 \%)$ & $1(100 \%)$ & $0(0.0 \%)$ & $0(0.0 \%)$ & $0(0.0 \%)$ & $0(0.0 \%)$ & $0(0.0 \%)$ & 0.00 \\
\hline & H2RA & $62(34 \%)$ & $24(39 \%)$ & $18(29 \%)$ & $5(8.1 \%)$ & $6(9.7 \%)$ & $5(8.1 \%)$ & $4(6.5 \%) * *$ & $1.39^{*}$ \\
\hline & [quarter/half/full] & {$[6 / 30 / 26]$} & {$[4 / 10 / 10]$} & {$[1 / 12 / 5]$} & {$[0 / 1 / 4]$} & {$[0 / 3 / 3]$} & {$[0 / 3 / 2]$} & [1/1/2] & [1.00/1.33/1.54] \\
\hline & PPI & $37(20 \%)$ & $20(54 \%) *$ & $8(22 \%)$ & $2(5.4 \%)$ & $3(8.1 \%)$ & $3(8.1 \%)$ & $1(2.7 \%)^{*}$ & $1.02 * *$ \\
\hline & [half/full] & {$[13 / 24]$} & {$[11 / 9]$} & {$[1 / 7]$} & {$[1 / 1]$} & {$[0 / 3]$} & {$[0 / 3]$} & {$[0 / 1]$} & {$[0.23 / 1.46]$} \\
\hline
\end{tabular}

Abbreviations:

L-ASA: low-dose aspirin, C: Cytoprotective gastric agents

H2RA: histamine type 2-receptor antagonists, PPI: proton pump inhibitors

PA: prostaglandin analogues, n/a: not applicable

MLS: modified Lanza score, $\quad{ }^{*} \mathrm{p}<0.05,{ }^{* *} \mathrm{p}<0.01$ compared with None, $\quad * * * \mathrm{p}<0.01$ compared with non-cessation 
(MLS $\geq 1$ ) in PPI users in the L-ASA cessation group was significantly lower than in patients receiving no prevention therapy ( $37 \%$ vs $64 \%$; $=0.004)$.

\section{Discussion}

In this retrospective study, $62 \%$ of patients receiving LASA had gastroduodenal mucosal lesions (MLS $\geq 1$ ), and $10 \%$ of patients had gastroduodenal ulcers. Niv et al reported in their prospective study that $48 \%$ of asymptomatic L-ASA users had gastroduodenal mucosal lesions (18). In Japanese patients, Nema et al reported that gastroduodenal mucosal lesions were found in $48.4 \%$ of patients with ischemic heart disease (19). Their reported prevalence rates of L-ASA-induced gastroduodenal mucosal lesions seem to be lower than in the present study. The difference in the prevalence rate might be due to differences in the candidate patients. In our study, about half of the L-ASA users had abdominal symptoms, including gastrointestinal bleeding signs. In this study, gastric mucosal lesions were significantly more observed than duodenal mucosal lesions $(61 \%$ vs $8.8 \%$; $\mathrm{p}=0.005$ ) in L-ASA users, consistent with a previous report (18). Although the frequency of gastric mucosal lesions in L-ASA users is high, it seems that there is no need to treat patients with mucosal injury of MLS=1-3 without symptoms. Occasionally, treatment may be necessary in patients with mucosal injury of MLS=4 to prevent aggregation.

We focused on gastroduodenal ulcers requiring treatment, and evaluated the factors associated with L-ASA-induced gastroduodenal ulcers in this study. Multiple logistic regression analysis revealed that a peptic ulcer history and the presence of abdominal symptoms were significant risk factors for L-ASA-induced gastroduodenal ulcers. A history of peptic ulcer was previously reported to be associated with a higher risk of upper gastrointestinal bleeding in L-ASA users (20). In this study we were not able to confirm the endoscopic findings of peptic ulcer scars in five patients who were considered to have a past history of peptic ulcer from the medical records. We speculate that the gastroduodenal ulcer scars had became unclear after healing, but we could not reject the possibility of erroneous history taking.

In regard to abdominal symptoms, a poor correlation is reported between the presence of epigastric symptoms and gastroduodenal ulcers (21); however, the significant association observed in this study between the presence of abdominal symptoms and L-ASA-induced gastroduodenal ulcers might be expected because the enrolled L-ASA users included many symptomatic patients with obvious bleeding signs.

In this study, the possible risk factors for L-ASA-induced gastroduodenal lesions included factors [i.e. advanced age (22), concomitant anticoagulants (23), concomitant antiplatelet drugs (11-13), concomitant NSAIDs (24) and concomitant corticosteroids (25) that have been previously reported to be associated with L-ASA-induced gastrointestinal complications. These five factors were not significantly associated with L-ASA-induced gastroduodenal ulcers in this study. Observational studies from Japan demonstrated that neither concomitant warfarin $(12,26)$ nor concomitant corticosteroids (26) was a significant risk factor for peptic ulcer in L-ASA users, which is consistent with our results. In this study, however, patients with two or more of these five factors were significantly associated with a higher prevalence of L-ASA-induced gastroduodenal ulcers, suggesting that summation of the risk factors is important for L-ASAinduced gastroduodenal ulcers.

As for concomitant NSAIDs, we speculated that NSAIDs were not regarded as significant risk factors in multiple regression analysis because of the small number of NSAIDco-prescribed L-ASA users in this study. In addition, 18 of 29 patients receiving NSAIDs were co-prescribed anti-ulcer drugs, which may have led to underestimation of the risk of NSAIDs. Multiple regression analysis in the subgroup with cytoprotective gastric agents or no gastric agents revealed that concomitant NSAIDs were a significant risk factor for gastroduodenal ulcers in L-ASA users [OR (CI): 5.47 (1.1126.92); $\mathrm{p}=0.037$ ].

Moreover, using multiple logistic regression analysis we identified the use of PPI, use of H2RA, and the cessation of L-ASA before endoscopy as preventive factors associated with L-ASA-induced gastroduodenal ulcers. A recent casecontrol study reported that both PPIs and H2RAs significantly reduced upper GI bleeding (27). In addition, the effectiveness of PPIs and H2RAs for the prevention of LASA-induced upper gastrointestinal mucosal injury was demonstrated in prospective randomized controlled trials $(28,29)$. In Japanese patients, the usefulness of PPIs and H2RAs was also reported in a previous observational study, case-control study and prospective cohort study $(12,30,31)$, consistent with our study; however, a prospective randomized controlled study is necessary to clarify the effectiveness of PPIs and H2RAs for the prevention of L-ASA-induced gastroduodenal injury in Japanese patients. In multiple regression analysis, we were not able to find a significant preventive effect of cytoprotective gastric agents on L-ASA induced gastroduodenal mucosal lesions. In addition, although the MLS in PPI plus cytoprotective gastric agents users was smaller than the MLS in PPI users, no significant additional effect of cytoprotective gastric agents was observed (0.86 vs 0.96 ; $\mathrm{p}=0.77$ in stomach, 0.14 vs $0.15 ; \mathrm{p}=0.69$ in duodenum).

L-ASA is often halted for three to seven days before endoscopy to decrease the risks associated with endoscopic procedures in Japanese patients. It is described in the Japanese guidelines for the diagnosis and treatment of gastric ulcer that cessation of NSAIDs is effective for NSAIDsinduced gastrointestinal injury (32); however, to our knowledge, no research has investigated whether L-ASA-induced gastroduodenal mucosal injury would improve with the short cessation of L-ASA, although it is reported that L-ASA induced gastroduodenal mucosal damage for a short period in 
healthy volunteers (33). In this study, the cessation of LASA for seven days was significantly associated with the lower prevalence of L-ASA-induced gastroduodenal ulcers. These results support the Japanese guidelines for the diagnosis and treatment of gastric ulcer. On the other hand, these results suggest that the gastroduodenal mucosal injury induced by L-ASA might be underestimated in patients with the cessation of L-ASA before endoscopy. This underestimation of L-ASA-induced gastroduodenal mucosal injury is thought to be clinically important, because it may prevent the prescription of gastric agents which should be prescribed in high-risk L-ASA users. In particular, when L-ASA users with two or more risk factors stop L-ASA administration before endoscopy, the endoscopic findings should be interpreted carefully.

In this study, significant differences between the L-ASA cessation group and the L-ASA non-cessation group were observed in the hospital in which patients were enrolled, suggesting that the policy for the cessation of L-ASA differed according to the hospital. Also, there were significantly fewer patients with obvious bleeding signs in the LASA cessation group than in the L-ASA non-cessation group, suggesting that there was no time to discontinue LASA because urgent endoscopy was necessary.

There are limitations to interpreting the results in this study. There might be a selection bias in the patients enrolled in this study because the data were collected from LASA users who had undergone upper gastrointestinal endoscopy rather than from all L-ASA users. Many enrolled patients $(47 \%)$ had abdominal symptoms. Moreover, the patients were registered from two hospitals and the disease severity of an underlying disease may have differed, although there was no difference in the rate of asymptomatic L-ASA users between the two hospitals. We are not able to deny the possibility that this bias affected the evaluation of risk factors for L-ASA-induced gastroduodenal mucosal lesions. Also, although medicine prescribed within one week before endoscopy was not considered a concomitant drug in this study, there was a difference in a prescription period of concomitant drugs by patients. This difference might have affected L-ASA-induced gastroduodenal mucosal lesions. As for concomitant PPI, there might be patients whose prescription period of concomitant PPI was not sufficient for healing gastroduodenal ulcers. And that might be the reason why PPI was not significantly associated with L-ASA-induced gastroduodenal ulcers in bivariate analysis. However, we were not able to evaluate the influence of the prescription period of a concomitant drug in this study. In addition, we did not evaluate Helicobacter pylori (HP) infection for LASA-induced gastroduodenal injury in this study because HP infection was investigated only in 47 patients (12\%). Since one of 18 HP-negative patients determined by tissue culture had been receiving PPI, this patient's HP-negative result was possibly a false-negative. While there is a report that HP infection might be a risk factor of L-ASA-induced gastroduodenal injury (34), there is another report that it might be a protective factor (35). The role of HP infection in L-ASA-induced gastrointestinal injury remains controversial. Also, we were not able to evaluate the extent to which the severity of an underlying disease influences L-ASAinduced gastroduodenal mucosal lesions because detailed data of the severity of an underlying disease were unknown in this study.

In summary, in this retrospective study, $10 \%$ of L-ASA users had gastroduodenal ulcers. A peptic ulcer history, the presence of abdominal symptoms and having two or more of five factors [i.e. advanced age $(\geq 75)$, concomitant anticoagulants, concomitant antiplatelet drugs, concomitant NSAIDs, and concomitant corticosteroids] were significant risk factors, and the use of PPI, use of H2RA, and the cessation of L-ASA before endoscopy were preventive factors for L-ASA-induced gastroduodenal ulcers. The cessation of L-ASA before endoscopy might lead to the underestimation of L-ASA-induced gastroduodenal injury.

\section{Acknowledgement}

This study was supported by a grant from the Global COE Program "Global Center of Excellence for Education and Research on Signal Transduction Medicine in the Coming Generation" from the Ministry of Education, Culture, Sports, Science, and Technology of Japan (T.A.). We thank Dr. I. Kamae for helpful comments on statistical analysis.

\section{References}

1. Collaborative overview of randomized trials of antiplatelet therapy. Maintenance of vascular graft or arterial patency by antiplatelet therapy. Antiplatelet Trialists' Collaboration. BMJ 308: 159-168, 1994.

2. Awtry EH, Loscalzo J. Aspirin. Circulation 101: 1206-1218, 2000.

3. Collaborative meta-analysis of randomized trials of antiplatelet therapy for prevention of death, myocardial infarction, and stroke in high risk patients. BMJ 324: 71-86, 2002.

4. Hayden M, Pignone M, Phillips C, Mulrow C. Aspirin for the primary prevention of cardiovascular events: a summary of the evidence for the U.S. Preventive Services Task Force. Ann Intern Med 136: 161-172, 2002.

5. Lauer MS. Aspirin for primary prevention of coronary events. $\mathrm{N}$ Engl J Med 346: 1468-1474, 2002.

6. Morimoto T, Nakayama M, Saito Y, Ogawa H. Aspirin for primary prevention of atherosclerotic disease in Japan. $\mathrm{J}$ Atheroscler Thromb 14: 159-166, 2007.

7. Nakayama M, Iwakiri R, Hara M, et al. Low-dose aspirin is a prominent cause of bleeding ulcers in patients who underwent emergency endoscopy. J Gastroenterol 44: 912-918, 2009.

8. Bhatt DL, Scheiman J, Abraham NS, et al. ACCF/ACG/AHA 2008 expert consensus document on reducing the gastrointestinal risks of antiplatelet therapy and NSAID use. Am J Gastroenterol 103: 2890-2907, 2008.

9. Sakamoto C, Sugano K, Ota S, et al. Case-control study on the association of upper gastrointestinal bleeding and nonsteroidal anti-inflammatory drugs in Japan. Eur J Clin Pharmacol 62: 765772, 2006.

10. Lanas A, Scheiman J. Low-dose aspirin and upper gastrointestinal damage: epidemiology, prevention and treatment. Curr Med Res Opin 23: 163-173, 2007.

11. Hallas J, Dall M, Andries A, et al. Use of single and combined antithrombotic therapy and risk of serious upper gastrointestinal 
bleeding: population based case-control study. BMJ 333: 726-728, 2006.

12. Shiotani A, Sakakibara T, Yamanaka Y, et al. Upper gastrointestinal ulcer in Japanese patients taking low-dose aspirin. J Gastroenterol 44: 126-131, 2009.

13. Sørensen R, Hansen M, Abildstrom S, et al. Risk of bleeding in patients with acute myocardial infarction treated with different combinations of aspirin, clopidogrel, and vitamin $\mathrm{K}$ antagonists in Denmark: a retrospective analysis of nationwide registry data. Lancet 374: 1967-1974, 2009.

14. Lanza FL, Royer GL, Nelson RS, Chen TT, Sechman CE, Rack MF. A comparative endoscopic evaluation of the damaging effects of nonsteroidal anti-inflammatory agents on the gastric and duodenal mucosa. Am J Gastroenterol 75: 17-21, 1981.

15. Robinson MG, Griffin JW, Bowers J, et al. Effect on ranitidine on gastroduodenal mucosal damage induced by nonsteroidal antiinflammatory drugs. Dig Dis Sci 34: 424-428, 1989.

16. Kobayashi K, Mizushima Y, Yanagawa A, et al. Prophylactic effects ranitidine, a $\mathrm{H} 2$ receptor antagonist, on gastric and duodenal lesions induced by nonsteroidal anti-inflammatory agents. J Adult Dis 20: 253-264, 1990 (in Japanese).

17. Naito $Y$, Yoshikawa $T$, Iinuma $S$, et al. Rebamipide protects against indomethacin-induced gastric mucosal injury in healthy volunteers in a double-blind, placebo-controlled study. Dig Dis Sci 43: 83-89, 1998.

18. Niv Y, Battler A, Abuksis G, Gal E, Sapoznikov B, Vilkin A. Endoscopy in asymptomatic minidose aspirin consumers. Dig Dis Sci 50: 78-80, 2005.

19. Nema $H$, Kato $M$, Katsurada $T$, et al. Investigation of gastric and duodenal mucosal defects caused by low-dose aspirin in patients with ischemic heart disease. J Clin Gastroenterol 43: 130-132, 2009.

20. Serrano P, Lanas A, Arroyo MT, Ferreira IJ. Risk of upper gastrointestinal bleeding in patients taking low-dose aspirin for the prevention of cardiovascular diseases. Aliment Pharmacol Ther 16: 1945-1953, 2002

21. Yeomans N, Lanas A, Talley N, et al. Prevalence and incidence of gastroduodenal ulcers during treatment with vascular protective doses of aspirin. Aliment Pharmacol Ther 22: 795-801, 2005.

22. Hernández-Díaz S, García Rodríguez LA. Cardioprotective aspirin users and their excess risk of upper gastrointestinal complications. BMC Med 4: 22, 2006.

23. Hurlen M, Abdelnoor M, Smith P, Erikssen J, Arnesen H. Warfarin, aspirin, or both after myocardial infarction. N Engl J Med
347: 969-974, 2002.

24. Sørensen HT, Mellemkjær L, Blot WJ, et al. Risk of upper gastrointestinal bleeding associated with use of low-dose aspirin. Am J Gastroenterol 95: 2218-2224, 2000.

25. Nielsen GL, Sørensen HT, Mellemkjœr L, et al. Risk of hospitalization resulting from upper gastrointestinal bleeding among patients taking corticosteroids: a register-based cohort study. Am J Med 111: 541-545, 2001.

26. Kawano K, Iizuka T, Yahagi N. Risk factor and preventive medicine about peptic ulcer by the low-dose aspirin. Ulcer Research 36: 44-48, 2009 (in Japanese).

27. Lanas A, García Rodríguez LA, Arroyo MT, et al. Effect of antisecretory drugs and nitrates on the risk of ulcer bleeding associated with nonsteroidal anti-inflammatory drugs, antiplatelet agents, and anticoagulants. Am J Gastroenterol 102: 507-515, 2007.

28. Yeomans N, Lanas A, Labenz J, et al. Efficacy of esomeprazole (20mg once daily) for reducing the risk of gastroduodenal ulcers associated with continuous use of low-dose aspirin. Am J Gastroenterol 103: 2465-2473, 2008.

29. Taha AS, McCloskey C, Prasad R, Bezlyak V. Famotidine for the prevention of peptic ulcers and oesophagitis in patients taking low-dose aspirin (FAMOUS): a phase III, randomized, doubleblind, placebo-controlled trial. Lancet 374: 119-125, 2009.

30. Nakashima S, Ota S, Arai S, et al. Usefulness of anti-ulcer drugs for the prevention and treatment of peptic ulcers induced by low doses of aspirin. World J Gastroenterol 15: 727-731, 2009.

31. Toyokawa $T$, Inaba $T$, Ishikawa $S$, Nakatsu $M$, Ando $M$, Tomoda J. Investigation of upper gastrointestinal bleeding after implantation of drug-eluting stents; prospective cohort study. Scand J Gastroenterol 45: 1097-1100, 2010.

32. EBM ni motozuku ikaiyou shinryou gaidorain dai2han. NSAID kaiyou. in EBM ni motozuku ikaiyou shinryou gaidorain. Jihou, 2007 (in Japanese).

33. Kawai $\mathrm{T}$, Yamagishi $\mathrm{T}$, Goto $\mathrm{S}$. Circadian variations of gastrointestinal mucosal damage detected with transnasal endoscopy in apparently healthy subjects treated with low-dose aspirin (ASA) for a short period. J Atheroscler Thromb 16: 155-163, 2009.

34. Pilotto A, Franceschi M, Longo MG, et al. Helicobacter pylori infection and the prevention of peptic ulcer with proton pump inhibitors in elderly subjects taking low-dose aspirin. Dig Liver Dis 36: 666-670, 2004

35. Hart J, Hawkey CJ, Lanas A, et al. Predictors of gastroduodenal erosions in patients taking low-dose aspirin. Aliment Pharmacol Ther 31: 143-149, 2010.

\footnotetext{
(C) 2010 The Japanese Society of Internal Medicine http://www.naika.or.jp/imindex.html
} 